\title{
Spectroscopy and terahertz imaging for sigillography applications
}

\section{P. Mounaix}

p.mounaix@loma.u-bordeaux.fr

\section{A. Younus}

\section{J.C. Delagnes}

\section{E. Abraham}

\section{Canioni}

\section{Fabre}

\author{
Laboratoire Ondes et Matière d'Aquitaine, Université de Bordeaux 1, UMR CNRS 5798, 351 Cours de \\ la libération, 33405 Talence Cedex, France \\ Laboratoire Ondes et Matière d'Aquitaine, Université de Bordeaux 1, UMR CNRS 5798, 351 Cours de \\ la libération, 33405 Talence Cedex, France \\ Laboratoire Ondes et Matière d'Aquitaine, Université de Bordeaux 1, UMR CNRS 5798, 351 Cours de \\ la libération, 33405 Talence Cedex, France \\ Laboratoire Ondes et Matière d'Aquitaine, Université de Bordeaux 1, UMR CNRS 5798, 351 Cours de \\ la libération, 33405 Talence Cedex, France \\ Laboratoire Ondes et Matière d'Aquitaine, Université de Bordeaux 1, UMR CNRS 5798, 351 Cours de \\ la libération, 33405 Talence Cedex, France \\ Centre de Recherche Bretonne et Celtique (EA 4451) Université de Bretagne Occidentale, 20 rue \\ Duquesne, 29238 Brest Cedex 3, France
}

Sigillography is the science that studies the manifold aspects of the seals. A seal can be defined as an imprint obtained on a malleable medium by imprinting an incised matrix, which transfers on it the characteristic signs of a person or an institution. We use THz spectroscopy and imaging for non-destructive evaluation of natural materials within old seals. Using a time domain THz spectroscopy and imaging system, THz transmission images are generated in the 0.1-3 THz range and demonstrate that the spectral properties of these materials allow to see across and under a thick layer of old materials. [DOI: 10.2971/jeos.2011.11002]

Keywords: terahertz, sigillography, non-destructive test, seals, bee wax, imaging

\section{INTRODUCTION}

Wax seals, (mainly of bee wax) formerly used to authenticate documents, are an attractive cultural, historical, and art research field. A seal is an imprint obtained by pressing an incised matrix onto a malleable medium (wax). This process transfers the characteristic stamp, signs or herald of a person or an institution. After being cooled down or ageing, these samples are very fragile. They tend to detach from their host documents over time. Although outmoded, seals were however one of the most common ways to proclaim the authenticity of a document in the latter Middle Ages. Emblematic figures, and habitually a name, surrounding it, identify the owner of the seal. Symbolically hair can sometimes be inserted in the seals and finger marks impressed on their back.

Their fragility is a crucial drawback to be overcome, to preserve this extraordinary historical source. For imaging these fragile artefacts, one can possibly use infrared, visible light and/or x-rays. Recently, terahertz time-domain spectroscopic (THz-TDS) imaging [1]-[4] has become an interesting and powerful tool for non-destructive testing and other applications. In the field of non-destructive testing and spectroscopic analysis, it is mandatory not only to visualize the samples but also to map its chemical composition [5], [6]. For this purpose, $\mathrm{THz}$ waves display attractive features such as good penetration depth, low scattering, free-space propagation, low photon energy, good beam coherence and broad spectral bandwidth. Since many materials exhibit spectral fingerprints in the $\mathrm{THz}$ region, spectroscopic analysis in this region has received much interest as an efficient and complementary tool for material characterizations. Furthermore, the availability of coherent optical imaging has made $\mathrm{THz}$ imaging an attractive non-contact, non-ionizing method for a variety of applications [7].

In this work, we characterize the dielectric response of several natural wax compounds in the terahertz spectral range [8]. Refractive index and absorption characteristics in the $\mathrm{THz}$ range are extracted and compared in order to discriminate different materials based upon original fingerprints or identical dielectric response over a huge frequency range. Due to low absorption level for certain wax materials, we will show the capabilities offered by $\mathrm{THz}$ spectroscopy and terahertz imaging with more realistic samples, demonstrating the availability to image under a thick seal layer.

\section{EXPERIMENT}

For the experiment, we use a standard THz-TDS transmission setup based on a mode-locked Ti-Sapphire laser providing 80 fs pulses with a $76 \mathrm{MHz}$ repetition rate. The laser output is split into pump and probe beams. The pump is fo- 


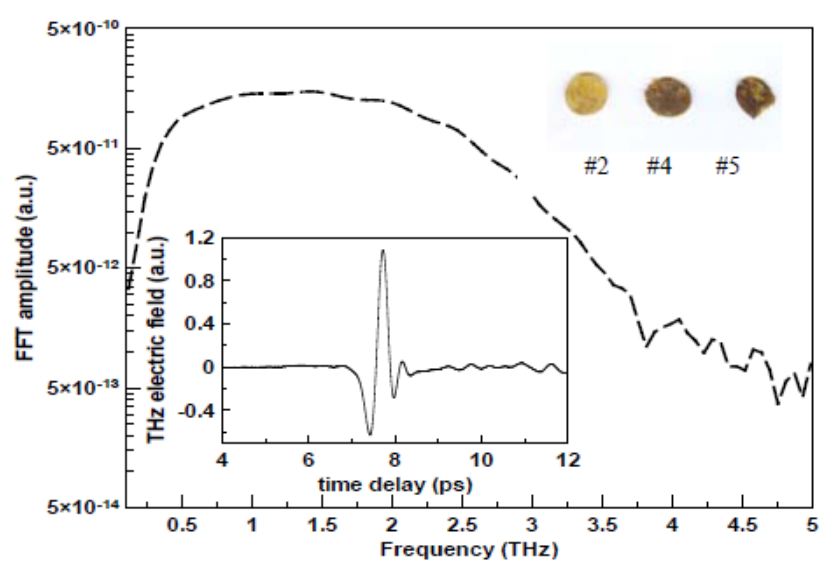

FIG. 1 Electric field waveforms recorded in the empty set-up (inset top). Corresponding spectrum (main). Inset: pellets of different waxes. The diameter is $10 \mathrm{~mm}$.

cused onto a bare InAs layer for surface field emission of the $\mathrm{THz}$ pulse which is further detected with a photo-switch triggered by the probe. After emission, the THz beam is collimated and focused at the sample's position. The timeresolved field variation is measured using the current generated in the LT-GaAs semiconductor receiver. The current induced by the probe beam in the detector is amplified and processed with a lock-in digital amplifier (SRS 830) referenced with a mechanical chopper located on the pump path. This set up provides a frequency response up to $3.5 \mathrm{THz}$.

Obtained with the impression of an incised matrix on a sufficiently malleable substance, the seal is made of a rather limited variety of materials, mainly poor materials. "Bee Sweat" is a natural (bees) wax extracted from the honeycomb in the beehives of Apis mellifera or Apis cerana (honey bees). Beeswax consists of a mixture of esters of fatty acids and fatty alcohols, hydrocarbons and free fatty acids. Data on the physical chemistry of waxes are complex and difficult to obtain. For example, the different colours of seals have been achieved by the addition of different metallic oxides. We can also found within the seals the addition of ancient fibres. Nevertheless, we propose to pragmatically analyze the $\mathrm{THz}$ dielectric responses of these waxes and wax mixtures. Then, we will present the dielectric properties of the basic materials of most of the seals, and we keep in mind that these results is an insight on the average values for extracted physical parameters and clearly the characterization of real old seals will probably gave different results due to the different technological process, materials, chemical reaction and ageing.

The first samples are made with cherry tree gum (sample \#1), bee wax from honey harvest in Brittany 1969 (sample \#2); honey harvest in Dordogne 2010 (sample \#3), the same sample after a slight thermal cycle typically $30^{\circ} \mathrm{C}$ (sample \#4), propolis (sample \#6) which is a resinous mixture that honey bees collect from tree buds, sap flows, or other botanical sources. It is used as a sealant for unwanted open spaces in the hive. Propolis is sticky at and above room temperature. We also measured some mixture of several waxes (\#5 and \#7).

Sample pellet preparation is made with a compact laboratory press. The maximum pressure of 8 tons ranges is applied for

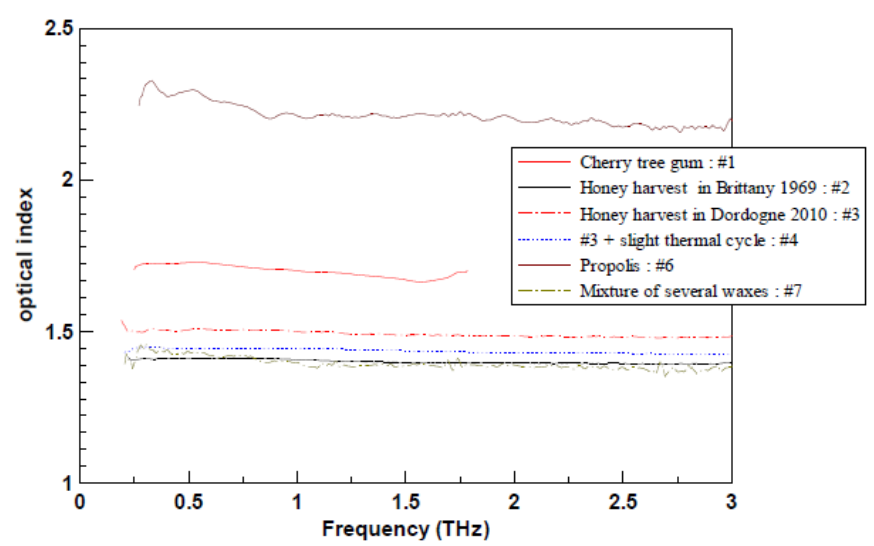

FIG. 2 Time dependence of the optical index as a function of the frequency.

the preparation of solid samples. The pellets produced are of extremely good quality. The thicknesses are ranging between 0.8 and $1.2 \mathrm{~mm}$ depending of the quantity of dyes and their viscosity. Then, the samples are chemically analysed by Light Induced Breakdown Spectroscopy (LIBS) technique and by Energy-dispersive X-ray spectroscopy (EDX).

These measurements reveal a major presence of carbon $(82 \%)$, oxygen $(14 \%)$ as expected and in sample \#1, a very significant quantity of calcium and a few percent of strontium.

\section{SPECTROSCOPY RESULTS}

For the derivation of the dispersion curve and the frequency dependence of the complex dielectric response, we carried out the Fast Fourier Transform of the time variations of the transmitted signal showed for example in Figure 1. In fact, a major advantage of this technique is that there is no need of a Kramers-Kronig analysis, since this experiment gives direct access to the electric field amplitude [9]. The time-domain Terahertz spectrometer allows unambiguously the determination of the complex dielectric response $\varepsilon^{*}(\omega)$.

It's possible to correlate the transmission function to the complex permittivity for thin samples. The multiple reflections due to finite sample thickness are taken into account. In the case of a homogeneous sample, the complex refractive index $N=n+i \kappa$ is related to the complex transmission function was numerically solved. To this aim; we measured the individual absorption spectra for the different samples. The results of the real part of the effective optical index are plotted in Figure 2 while Figure 3 represented the absorption variations (plotted here between 0.2 and $3 \mathrm{THz}$ ). For all the materials under investigation, the dispersion of the refractive index values is quite flat all over the terahertz frequency range with data around 1.4-1.6 which is in good agreement with optical measurements [10].

These preliminary results demonstrate that no typical fingerprints are found for the beeswax compounds. In Figure 3, it's clear that they all exhibit a gradual increasing trend with frequency as expected for most amorphous materials. The 


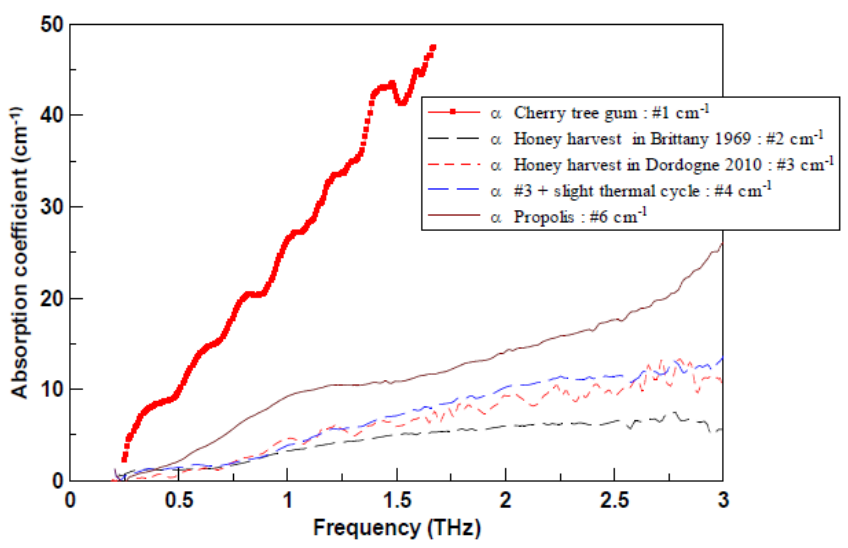

FIG. 3 Frequency dependence of the absorption coefficient for different type of waxes.

highest rate of increasing opacity was displayed by the propolis and cherry gum samples, and the lowest rate was displayed by the bee wax materials. Nevertheless, since it was very difficult to discriminate quantitatively or even qualitatively the intrinsic difference of these materials, the clear difference value of the optical index for samples \#1 (cherry gum) and \#6 (propolis) is a welcome feature to identify these different waxes content by time domain spectroscopy or imaging. To predict the attenuation of the overall transmission through the materials, we can model it in a first approximation consider a constant frequency dependant attenuation constant [11] or an absorbance spectrum fitting method [12]. Due to the complexity (lot of chemical components) of this amorphous material, these simple models fail in the prediction of the absorption dispersion in the $\mathrm{THz}$ range. Nevertheless, as a first characterization, it's prime of importance to prebuilt a library of reference data in order to propose a fitting absorbance for identification method. Another interesting possibility is related to scattering [13] for surface texture of the air-soft materials at room temperature; roughness in the range of $\mathrm{THz}$ wavelength could be important.

\section{NON-INVASIVE IMAGING}

Since the $\mathrm{THz}$ transmits through the seal and enables one to non-destructively evaluate the interior structure, the $\mathrm{THz}$ image could show the presence of a larger number of peculiar materials that would nominally be invisible if one were to simply visibly inspect the seal surfaces correlated to the attenuation of the transmitted $\mathrm{THz}$ signal. $\mathrm{THz}$ images can also be generated based on the time delay in the arrival of the $\mathrm{THz}$ pulse or analogously the frequency dependent phase of the $\mathrm{THz}$ wave. In a previous work [14], far infrared transmission spectra of several graphite pencil mines have been measured in the terahertz range using time-domain spectroscopy. The observation of the gradual absorption depending on the graphite proportion has been demonstrated for different pencils from hard to soft black-marking graphite mines. The resulting graphite transmittance is used to perform two-dimensional transmission terahertz imaging of
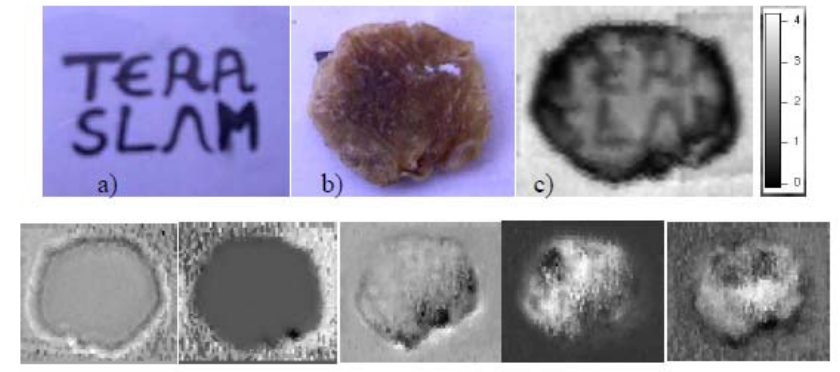

$20 \mathrm{ps}$

20.5 ps ,

$21 \mathrm{ps}$,

21.5ps,

$22 \mathrm{ps}$,

FIG. 4 Optical view of the logo (a), the wax onto the paper; the diameter is $25 \mathrm{~mm}$ (b), time amplitude of the transmitted THz pulse (c), several time delay images with an arbitrary scale for the false colour (d)

written documents. Figure 4 represents the optical pictures of the name of our group written onto a sheet of paper (Figure $4 \mathrm{a}$ ) with a $4 \mathrm{H}$ lead, the word recovered by a thick layer of sample \#4 (Figure $4 \mathrm{~b}$ ) and the images deduced from the time-amplitude of $\mathrm{THz}$ signal in transmission (Figure 4c). Since the arrival time is determined by the optical path length (i.e., the product of the real index of refraction and the physical thickness of the sample over each pixels), one would demonstrate in Figure $4 \mathrm{~d}$ that the contrast in a time delay or phase image which is the result of either local variations in the thickness of the sample or variations in the real index of refraction is not suitable in this case or inhomogeneous materials. Each picture can be considered as a sort of local cutting inside the wax sample, slice by slice.

More precisely, Figure $4 \mathrm{c}$ is obtained by selected the max amplitude of the transmitted electric field, the maximum signal mode is then correlated to the average absorption over the frequency bandwidth. In Figure $4 d$, we provided different time delay obtained every 0.5 ps. So the contrast was given by approximately the average refractive index integrated over the thickness at a given pixel. So, that means this purpose of imaging objects in constant-time mode is difficult to interpret in the case of an inhomogeneous material. In both images, the dark edge around the samples is caused by diffraction, stronger scattering or absorption due to a thickness difference. Nevertheless, we can observe that the thin layer of lead does not affect the image when we selected the optical thickness as a contrast parameter. Concerning the roughness of the surface, we can estimate that we will be able to discriminate a thickness of $30 \mu \mathrm{m}$ due to temporal precision of phase delay [14].

The resulting graphite transmittance mimics the response of old inks with metal contents and is then used in our case to perform two-dimensional transmission terahertz imaging of possible written documents under this thick seal layer. Finally, we believe on the real advantage of the terahertz imaging apparatus which could be powerful for the investigation of old underwriting beneath wax layers or foreign objects inserted into the seal. For example, our future works will be focus on medieval inks, such as sour galls, aleppo galls, green and blue vitriol. 
Finally, we believe in the real advantage of the terahertz imaging apparatus which could be powerful for the investigation of old underwriting beneath wax layers [15] or foreign objects inserted into the seal.

\section{CONCLUSION}

In summary, THz imaging is applied to non-destructive evaluation of original seals. Using a time domain $\mathrm{THz}$ spectrometer and imaging system, $\mathrm{THz}$ transmission images are generated in the $0.1-3 \mathrm{THz}$ range. The presence of defects or objects in the seals will be evident in the $\mathrm{THz}$ image due to enhanced scattering or absorption of the $\mathrm{THz}$ radiation. Pragmatic analysis of the spectral properties of these materials shows that the $\mathrm{THz}$ absorbance is sufficient low to analyze spectral properties of foreign inclusion and see under a thick layer of old basic materials in relation to official written documents. The study, the appreciation and, in particular, the preservation and restoration of the seals will interest and propose a tool to preserve old archives, and participate to this immense vast cultural patrimony.

\section{References}

[1] B. Hu, and M.C. Nuss, "Imaging with terahertz waves" Opt. Lett. 20, 1716-1718 (1995).

[2] D. Mittleman, S. Hunsche, L. Boivin, and M.C. Nuss, "T-ray tomography“ Opt. Lett. 22, 904-906 (1997).

[3] B. Ferguson, S. Wang, D. Gray, D. Abbot, and X.-C. Zhang, "T-ray computed tomography" Opt. Lett. 27, 1312-1314 (2002).

[4] A. El Fatimy, J.C. Delagnes, A. Younus, E. Nguema, F. Teppe, W. Knap, E. Abraham, and P. Mounaix, "Plasma wave field effect transistor as a resonant detector for 1 terahertz imaging applications" Opt. Commun. 282, 3055-3058 (2009).

[5] K. Kawase, Y. Ogawa, Y. Watanabe, and H. Inoue, "Nondestructive terahertz imaging of illicit drugs using spectral fingerprints" Opt. Express 11, 2549-2554 (2003).
[6] Y.C. Shen, T. Lo, P.F. Taday, B.E. Cole, W.R. Tribe, and M.C. Kemp, "Detection and identification of explosives using terahertz pulsed spectroscopic imaging“ Appl. Phys. Lett. 86, 241116 (2005).

[7] E. Abraham, A. Younus, J.C. Delagnes and P. Mounaix, "Noninvasive investigation of art paintings by terahertz imaging" Appl. Phys A 100, 585-590 (2010).

[8] C. Roman, 0. Ichim, L. Sarger, V. Vigneras, and P. Mounaix, "Terahertz dielectric characterisation of polymethacrylimide rigid foam: the perfect sheer plate?" Electron. Lett. 40, 1167-1169 (2004).

[9] P. Mounaix, M. Moustakim, S. Le Boiteux, J. P. Delville, R. Wunenburger, and L. Sarger, "Far-infrared optical constants of $\mathrm{CO}_{2}$ near the critical point measured by terahertz spectroscopy" Appl. Phys. Lett. 83, 5095-5097 (2003).

[10] M.C. Waller, M.A.Seibert, "Studies of refractive indices of binary wax mixtures" The american oil chemist'society 32, 709-712 (1947).

[11] J.E. Bjarnason, T.L.J. Chan, A.W.M. Lee, M.A. Celis, and E.R. Brown, "Millimeter-wave, terahertz, and mid-infrared transmission through common clothing" Appl. Phys. Lett. 85, 519-521 (2004).

[12] Y. Wang, Z. Zhao, Z. Chen, K. Kang, B. Feng, and Y. Zhang, "Terahertz absorbance spectrum fitting method for quantitative detection of concealed contraband" J. Appl. Phys. 102, 113108 (2007).

[13] L. Zurk, B. Orlowski, D. Winebrenner, E. Thoros, M. Leahy-Hoppa, and L. Hayden, "Terahertz scattering from granular material" J. Opt. Soc. Am. B 24, 2238-2243 (2007).

[14] E. Abraham, A. Younus, A. El Fatimy, J.C. Delagnes, E. Nguéma, and P. Mounaix, "Broadband terahertz imaging of documents written with lead pencils" Opt. Commun. 282, 3104-3107 (2009).

[15] K. Fukunaga, Y. Ogawa, S. Hayashi, and I. Hosako, "Terahertz spectroscopy for art conservation" IEICE Electron. Express 4, 258263 (2007). 